\section{Linfocitos TCD8+ autorreactivos en pacientes con leucemia mieloide crónica en asociación con HLA e infección con adenovirus}

Sergio E Rivera, ${ }^{1,2}$ Miriam Echeverría, ${ }^{1}$ Pedro Salcedo, ${ }^{2}$ Georgina Márquez, ${ }^{2}$ Zuhey Carrillo, ${ }^{1}$ Yennis Parra, ${ }^{1}$ Ana María Cipriani, ${ }^{1}$ José R. Núñez, ${ }^{1}$ Melchor Álvarez de Mon, ${ }^{2}$ Atilio Farruco ${ }^{1}$

\section{Resumen}

ANTECEDENTES: algunos adenovirus se han señalado como activadores clonales en leucemias. El alelo HLA-DRB1* 14 subtipos DRB1*14:21, $14: 22,14: 45,14: 26,14: 33,14: 51,14: 35$ se asociaron con leucemia mieloide crónica (LMC) en pacientes venezolanos.

OBJETIVO: evaluar el mimetismo molecular entre el adenovirus y la estructura del antígeno HLA-DRB1*14 que exhiben el mismo cambio en la posición de aminoácido del epítopo DR53.

MATERIAL Y MÉTODO: estudio experimental realizado en el IHO Banco de Sangre del Estado Zulia, Venezuela en muestras de sangre periférica de pacientes con LLA, LMC y controles sanos. Se realizaron cultivo mixto de linfocitos, serología, proliferación linfocitaria y citofluorometría.

RESULTADOS: los linfocitos DRB1*14 del paciente reaccionaron en 48 horas versus los linfocitos DRB1*14 estimuladores, que exhibieron aumento de los linfocitos T CD8+. Los pacientes con LMC tuvieron un perfil serológico diferente contra el adenovirus. Sólo pacientes con LMC reaccionaron frente al péptido secuencia LLERRRA con incremento de las células TCD8+.

CONCLUSIÓN: se estableció que la relación leucemia mieloide crónica, HLA-DRB1*14, células TCD8+ de memoria autorreactivas y TCD8+ en respuesta específica frente al adenovirus podría estar en el origen de la leucemia mieloide crónica de pacientes venezolanos.

PALABRAS CLAVE: leucemia linfoide aguda (LLA), leucemia mieloide crónica (LMC), TCD8+, HLA, adenovirus, autoinmunidad

\footnotetext{
${ }^{1}$ Universidad del Zulia, Maracaibo, Venezuela. 2 Instituto Hematológico de Occidente, Banco de Sangre del estado Zulia, Maracaibo Venezuela.
}

Recibido: 16 de noviembre 2015

Aceptado: 2 de marzo 2016

Correspondencia Dr. Sergio E. Rivera Av. 3F con calle 86, Edifico Luxus I, piso 3, Apto 3C, Maracaibo, estado de Zulia, Venezuela. sergio.rivera54@hotmail.es

Este artículo debe citarse como Rivera SE, Echeverría M, Salcedo P, Márquez G, Carrillo Z, Parra Y, Cipriani AM, Núñez JR, Álvarez de Mon M, Farruco A. Linfocitos TCD8+ autorreactivos en pacientes con leucemia mieloide crónica en asociación con HLA e infección con adenovirus. Rev Alerg Méx. 2016 abr-jun; 63(2):163-168. 


\section{Autoreactive TCD8+ lymphocytes in patients with chronic myeloid leukemia in association with HLA and adenovirus infection}

Sergio E Rivera, ${ }^{1,2}$ Miriam Echeverría, ${ }^{1}$ Pedro Salcedo, ${ }^{2}$ Georgina Márquez, ${ }^{2}$ Zuhey Carrillo, ${ }^{1}$ Yennis Parra, ${ }^{1}$ Ana María Cipriani, ${ }^{1}$ José R. Núñez, ${ }^{1}$ Melchor Álvarez de Mon, ${ }^{2}$ Atilio Farruco ${ }^{1}$

\begin{abstract}
BACKGROUND: A possible interaction between a specific HLA type and Adenovirus has been postulated as a promoter in leukemia clonal evolution. The HLA-DRB1*14, specifically DRB1*14:21, 14:22, 14:45, $14: 26,14: 33,14: 51,14: 35$ subtypes was the most frequent in CML venezuelan patients.
\end{abstract}

OBJECTIVES: It is interesting to evaluate the molecular mimicry between the Adenovirus and the DRB $1 * 14$ subtypes which exhibit the same change in the amino acid position of the DR53 epitope. This mimicked segment has been identified as a LLERRRA polypeptide.

MATERIAL AND METHODS: Experimental research conducted in the IHO Venezuela, in peripheral blood samples of patients with ALL, CML and healthy controls. Mixed culture, serology, lymphocyte proliferation and cytofluorometry were performed.

RESULTS: DRB1*14 patient's lymphocytes reacted in 48 hours mixed culture against DRB $1 * 14$ promoters lymphocytes exhibiting increased CD8+T lymphocytes. CML patients show a different serological profile against Adenovirus. Only CML patients reacted to LLERRRA peptide, increasing CD8+ T cells.

CONCLUSION: It is established that the relationship CML, HLADRB1*14, autoreactive CD8+ T memory cell and CD8+T specific response from Adenovirus could be at the origin of the $C M L$ in venezuelan.

KEYWORDS: Leukemias, TCD4+, TCD8+, Adenovirus, HLA association and disease

\footnotetext{
${ }^{1}$ Universidad del Zulia, Maracaibo, Venezuela.

${ }^{2}$ Instituto Hematológico de Occidente, Banco de Sangre del estado Zulia, Maracaibo Venezuela.
}

\section{Correspondence}

Dr. Sergio E. Rivera

Av. 3F con calle 86, Edifico Luxus I, piso 3, Apto 3C,

Maracaibo, estado de Zulia, Venezuela. sergio.rivera54@hotmail.es

\section{ANTECEDENTES}

La leucemia mieloide crónica es una mieloproliferación clonal causada por la transformación neoplásica de una célula madre pluripotencial, caracterizada por una llamativa hiperproducción de granulocitos. Pareciera claro que la mayor parte de las leucemias en los seres humanos no son causadas por retrovirus oncogénicos, sino por mutaciones aleatorias. Sin embargo, el estudio de retrovirus que causan leucemia en animales señala claramente la existencia de un mecanismo por el cual los genes pueden ser más comúnmente mutados. ${ }^{1,2}$

La asociación de esta enfermedad neoplásica con el complejo principal de histocompatibilidad $(\mathrm{CPH})$ se origina de los estudios en el virus 
de Gross, inductor de la leucemia múrida, en los que se observó que los ratones con el haplotipo $\mathrm{H}-2^{\mathrm{k}}$ sucumbían más rápidamente a la leucemia que los ratones con el haplotipo $\mathrm{H}-2^{\mathrm{d}}$. La asociación del alelo DRB1* y la leucemia mieloide crónica se evaluó en pacientes japoneses y venezolanos con varias asociaciones positivas y negativas, coincidiendo con la asociación positiva DRB1*14 y la leucemia mieloide crónica. ${ }^{3}$ En la población venezolana se describió el alelo HLA-DRB1*14, específicamente los subtipos DRB1*14:21, 14:22, 14:45, 14:26, 14:33, 14:51, 14:35, de riesgo para la leucemia mieloide crónica en venezolanos. ${ }^{4}$ Esta observación es de particular interés debido al mimetismo mostrado por varios virus oncogénicos para el epítope HVR3 del DR53 que se extiende a los alelos DRB1*01, DRB1*10 y DRB1*14.5,6 En este caso en particular interesa el mimetismo molecular entre el adenovirus y los subtipos del alelo DRB1*14 (1401, 1407, 1408, 1410, 1411, 1414, 1418, 1423,1426, 1428, 1431, 1432, $1434,1435,1436,1438$ y 1439), los cuales tienen el mismo cambio de posición observado en el aminoácido del epítope DR53.5,7 Este segmento mimetizado se ha identificado como un polipéptido de 7 aminoácidos de secuencia LLERRRA según los trabajos realizados por Dorak y Matsuoka en 1996. ${ }^{5,7}$ Es de acotar también que existen varios reportes que indican una asociación entre $\mathrm{t}(9 ; 22)(\mathrm{q} 34 ; \mathrm{q} 11)$ y diferentes alelos HLA. Esta asociación sugiere un posible papel de las células T citotóxicas en la patogénesis de la enfermedad relacionado con las proteínas de fusión bcr-abl.8,9

Ninguna neoplasia humana ha sido inequívocamente asociada con los adenovirus, más aún, no se ha probado una asociación de las leucemias con procesos infecciosos causados por virus. ${ }^{10}$ Se ha propuesto una posible interacción entre un tipo específico de HLA y tipos específicos de adenovirus como promotora en la evolución clonal de la leucemia linfoblástica aguda de linaje B. ${ }^{5}$ Pero además, podría plantearse, con base en el marcado tropismo hacia las células B, sus características inmuno-evasivas, su mimetismo molecular con HLA DR53 y el hecho que el alelo HLA DRB $1 * 14$ muestra el mismo cambio de posición observado en el aminoácido del epítope DR53, que las infecciones tempranas con adenovirus representarían un riesgo relativo de generar leucemia mieloide crónica. ${ }^{4,5}$

\section{MÉTOdOS}

Es un estudio experimental, realizado en el Instituto Hematológico de Occidente (IHO), Banco de Sangre del Estado Zulia, Venezuela, Laboratorio de HLA Inmunología. Se evaluaron muestras de sangre periférica de pacientes con LLA, LMC y controles sanos quienes expresaron por escrito su consentimiento. Para la determinación serológica de anticuerpos $\operatorname{lgG}$ e $\lg \mathrm{M}$ a adenovirus en plasma se tomaron 8 pacientes con leucemia mieloide crónica, 10 pacientes con LLA y cinco controles sanos. La determinación IgM e IgG para adenovirus se realizó con ELISA de fase sólida para determinación de anticuerpos (IgM e IgG) marca Laboratorio DRG Instruments GMBH, DRG products Ref. EIA 3447. Los controles $4 / 5$ y pacientes LLA 9/10, solo mostraron IgM+. Los pacientes con leucemia mieloide crónica resultaron 2/8 IgM+, $1 / 8 \lg \mathrm{G}+$, el resto $5 / 8$ negativos.

La suspensión de linfocitos de $1 \mathrm{~mL}$ a $2 \times 10^{6}$ \% $\mathrm{mL}$ en RPMI 1640 + suero de ternera fetal (STF) al 5\% más penicilina-estreptomicina (respondedores) se enfrentó con $1 \mathrm{~mL}$ de linfocitos a $4 \times 10^{6} / \mathrm{mL}$ en RPMI 1640 más suero de ternera fetal (STF) al 5\% más penicilina-estreptomicina tratados con mitomicina (mitomicna sigma) a una concentración de $4 \mu \mathrm{g} / \mathrm{mL}$ durante 2 horas (estimuladores) en frascos Falcon, cultivadas en atmósfera húmeda de $5 \%$ de $\mathrm{CO}_{2}$ a $37^{\circ} \mathrm{C}$ durante 48 horas. Como respondedores se utilizaron: un control HLA-DRB1*08-11 y un paciente con 
leucemia mieloide crónica HLA-DRB1*14-04. Como estimulador se utilizó un control sano HLA-DRB1*13-14 tratado con mitomicina. Se evaluaron por citometría de flujo, marcadas con anticuerpos monoclonales anti-CD8, anti CD4 y anti-CD3 (Beckman Coulter,USA) alícuotas de $200 \mu \mathrm{L}$ de la mezcla respondedor-estimulante, a tiempo 0 (T0) y otra a las 48 horas (T48).

\section{RESULTADOS}

El porcentaje de células TCD8+ aumentó de $5.54 \%$ (T0) a $18.9 \%$, solo al enfrentar el paciente con leucemia mieloide crónica HLA-DRB $1 * 14-04$ con el control mitomizado HLA-DRB1*13-14 a las 48 horas de cultivo (T48), típico de células memoria (Cuadro 1). Otro paciente con leucemia mieloide crónica tipificado HLA-DRB1*14-13 se encontró en estado blástico al momento de la toma de la muestra y no fue incluido en el cultivo. Sin embargo, al examen citofluorométrico mostró $25 \%$ de células marcadas con el TCD4+ y $41.3 \%$ marcadas con el TCD8+ y un índice CD4/CD8 de 0.61, totalmente diferente al observado en controles.

Se cultivaron linfocitos de sangre periférica obtenidos de: control 1: individuo sano HLA DRB1*08-11, IgG e IgM anti adenovirus negati-

Cuadro 1. Porcentaje de positividad en citofluorometría para los marcadores linfocitarios CD3, CD4, CD8 e índices CD4/ CD8 de controles y un paciente LMC en cultivo mixto de linfocitos frente a dianas HLA-DRB1* y citometría de flujo en pacientes con LMC y controles sanos

$\begin{array}{lcccc}\begin{array}{l}\text { Cultivo } \\ \text { Marcador }\end{array} & \mathbf{1 - 3 M / T 0} & \mathbf{2 - 3 M} / \mathbf{T 0} & \mathbf{1 - 3 M} / \mathbf{T 4 8} & \mathbf{2 - 3 M} / \mathbf{T 4 8} \\ \text { \%CD3 } & 38,2 & 44,6 & \mathrm{ND} & \mathrm{ND} \\ \text { \%CD4 } & 28,5 & 38,5 & 26,1 & 28,8 \\ \text { \%CD8 } & 6,58 & 5,54 & 6,76 & 18,9 \\ \text { CD4/CD8 } & \mathbf{4 , 3} & \mathbf{6 , 9 5} & \mathbf{3 , 8 6} & \mathbf{1 , 5 2}\end{array}$

1 = Control DRB $1 * 08-11 ; 2$ = Paciente LMC DRB $1 * 14-13$; $3 \mathrm{M}=$ Control DRB $1 * 14-04$ tratado con mitomicina (Estimulante); $\mathrm{T} 0=$ Tiempo de 0 horas; $\mathrm{T} 48=$ Tiempo de 48 horas. Los índices CD4/CD8 fueron expresados en valores absolutos. vo; control 2: individuo sano HLA DRB1*14-13, IgM+ anti adenovirus; paciente 1 : individuo con leucemia mieloide crónica, HLA DRB1*16-03 y IgM e IgG anti adenovirus negativos y paciente 2 : individuo con leucemia mieloide crónica, HLA DRB1*14-04 IgG e IgM adenovirus negativo. Se resuspendieron dos mililitros de cada suspensión linfocitaria de todas las muestras de este estudio a $1.5 \times 10^{6} / \mathrm{mL}$ en medio RPMI 1640 suplementado con suero fetal de ternera (SFT) al 10\% más penicilia-estreptomicina y se colocaron en frascos Falcon. A cada frasco se le añadieron 25 microgramos del péptido LLERRRA (GenScript, 98,3\% de pureza, NJ,USA, la concentración seleccionada del péptido fue calculada en base a un estudio de dosis respuesta) y 330 microgramos de interleucina 2 (IL-2 RD System; UK), concentración óptima recomendada por el fabricante. Los linfocitos se cultivaron en atmósfera húmeda de $\mathrm{CO}_{2}$ al $5 \%$ por espacio de 120 horas (cinco días); posteriormente se marcaron con anticuerpos monoclonales antiCD8, anti CD4 y anti-CD3 alícuotas de $200 \mu \mathrm{L}$ de cada suspensión. Paralelamente se cultivaron los mismos pacientes y controles en la seroalbúmina humana $(\mathrm{SAH})$ en lugar del péptido LLERRRA y se evaluaron de la misma manera alícuotas de $200 \mu \mathrm{L}$ de cada una en citometría de flujo, antes descritas.

Después de la estimulación con el péptido LLERRRA del adenovirus, el porcentaje de TCD3+ aumentó en ambos controles; de $49 \%$ pasó a $64 \%$ para el control 1 y de 31 a $57 \%$ para el control 2 . Para los pacientes, el aumento fue de 46 a $80 \%$ para el paciente 1 y de 34 a $57 \%$ para el paciente 2 .

En cuanto al marcador TCD8+, los controles 1 y 2 se mostraron levemente disminuidos; para el control 1 los porcentajes variaron de 18 a $16.7 \%$ y para el control 2 esta variación fue de 10 a $9 \%$. Por el contrario, en los pacientes 1 y 2 estimulados con el péptido LLERRRA del adenovirus, el marcador TCD8+ aumentó de 17 a 24\% en el paciente 1 y de 8 a $14 \%$ en el paciente 2 . 
Para los controles y los pacientes se observó una proliferación importante en la población TCD4+. El aumento fue de 25 a $42 \%$ para el control 1 , de 17 a $48 \%$ en el control 2 , de 23 a $55 \%$ en el paciente 1 y de 19 a $42 \%$ en el paciente 2 (Figura 1 ).

\section{DISCUSIÓN}

Existe la posibilidad de que una respuesta contra el epítope HVR3 del virus podría conducir, por reactividad cruzada, a una respuesta autoinmunitaria contra la estructura antigénica del DRB1*14. En ambos casos la determinación serológica de anticuerpos contra adenovirus muestra una pobre respuesta humoral en pacientes con leucemia mieloide crónica. La determinación de linfocitos TCD8+ contra el epítope HVR3 permitió valorar los mecanismos

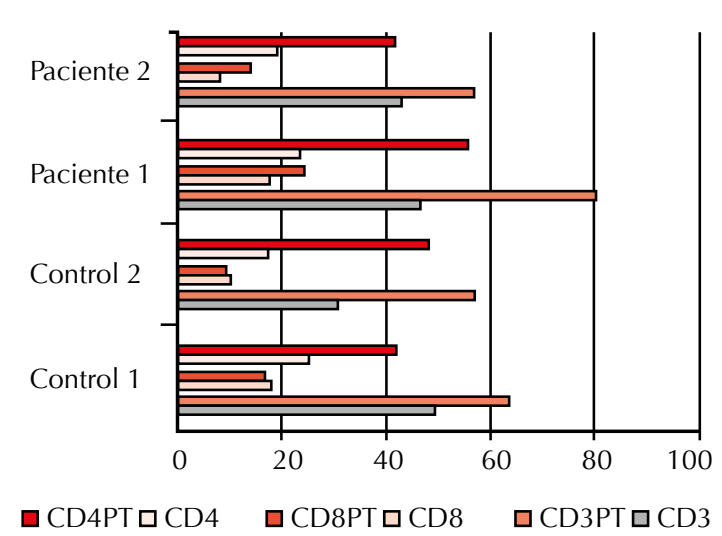

Control 1: HLA DRB1*08-11, IgG e IgM adenovirus negativo; Control 2: HLA DRB1*14-13, IgM adenovirus positivo; Paciente 1: LMC HLA DRB1*16-03, IgM e IgG adenovirus negativo; Paciente 2: LMC HLA DRB1*14-04, $\lg \mathrm{G}$ e IgM adenovirus negativo

Figura 1. Representación gráfica de la citometría de flujo de cultivo linfocitario de controles y paciente LMC estimulados con el péptido viral de secuencia LLERRRA del adenovirus. Las columnas marcadas con "PT" representan la estimulación frente al péptido. Los resultados se expresan en porcentajes para cada uno de los marcadores CD3, CD4 y CD8 medidos en citometría de flujo. inmunitarios celulares antivirales en controles sanos y pacientes con leucemia mieloide crónica, que mostró que existe una respuesta TCD8+ memoria en pacientes con leucemia mieloide crónica que pudo detectarse solo al enfrentarlos en cultivo mixto a individuos DRB1*14. La respuesta proliferativa de pacientes con leucemia mieloide crónica frente al péptido LLERRRA del adenovirus a diferencia de los controles muestra una respuesta TCD8+ y no TCD4+.

Invitamos a laboratorios con mejores recursos a replicar esta investigación. La estricta selección y la dificultad de contar con pacientes leucémicos no tratados encarecen enormemente este trabajo. Sin embargo, la fuerte asociación positiva HLA-DRB1*14 con las leucemias mieloides crónicas, la generación de células TCD8+ memoria exclusiva anti DRB1*14 y la reacción proliferativa TCD8+ frente al péptido LLERRRA del adenovirus en individuos serológicamente negativos, se encuentran a favor de un mecanismo de respuesta inmunitaria con un evidente componente autoinmune, al origen de las leucemias mieloides crónicas en pacientes venezolanos.

\section{Agradecimientos}

Subvencionado por el Proyecto CONDES No. CC-0187-11 y el Instituto Hematológico de Occidente, Maracaibo, Estado Zulia, Venezuela

\section{REFERENCIAS}

1. Smith KA, Griffin JD. Following the cytokine Signaling pathway to leukemogenesis: a chronology J Clin Invest. 2008 Nov;118(11):3564-73.

2. Perrotti O, Jamieson C, Goloman J, Skorski T. Chronic myeloid leukemia: mechanisms of blastic transformation J Clin Invest. 2010; 120(7):2254-64.

3. Yasukawa $\mathrm{M}$, Ohminami $\mathrm{H}$, Kojima K, Inokuchi K, Nishimura Y, Fujita S. Analysis of HLA-DRB1 alleles in Japanese patients with chronic myelogenous leukemia. Am J Hematol. 2000; 63(2):99-101. 
4. Rivera S,Echeverria M, Marquez G, Villalobos C.C, Pereira N, DE Salvo L, Carrillo Z, Parra Y. Asociación HLA-DR*/ DQ*/DPA1* con leucemias linfoides agudas (LLA) y leucemias mieloides crónicas (LMC) en la población mestiza del estado Zulia, Venezuela (Estudio Preliminar). Ciencia 2007;15(4):365-372.

5. Dorak M. Frequently asked questions about HLA-DR53 and LEUKEMIA HLA-related LINKS SNP analysis of HLA-DRB4 entrez-gene: HLA-DRB4 dbSNP: HLA-DRB4Uni Gene- HLADRB4: Hs.696211 protein database: HLA-DRB4. Blog Last updated on 25 February 2008. Address for bookmark: http://www.dprak.info/hla/hla-dr53.html

6. Schlehofer B, Blettner M, Geletneky K. Sero-epidemiological analysis of the risk of virus infections for childhood leukemia. Int J Cancer. 1996;65:584-590
7. Matsuoka M, Hattorl T, Nishimura Y, Takatsuki K. ATL cells recognize self class II HLA antigens: Implication to leukemogenesis. Leukemia 1995;8:1338-43.

8. Yasukawa M, Ohminami H, Kojima K, Hato T, Hasegawa A, Takahashi T, Hirai H, Fujita S. HLA class II-restred antigen presentation of endogenus bcr-abl fusión protein by chronic myelogenus leukemia-derived dendritic cells to $C D 4+T$ lymphocytes. Blood 2001;98(5):1498-1505.

9. Mundhada S, Luthra R, Cano P. Association of HLA Class I and Class II genes with bcr-abl transcripts in leukemia patients with $\mathrm{t}(9 ; 22)$ (q34; q11) BMC Cancer 2004;4:25. This article is available from: http://www.biom dcentral. com/:1471-2407/4/25.

10. Hunt K, Vorburgers S, Suisher S. Ed. 2007 Humana Press Inc. Gene Therapy for Cancer 